\title{
Nuclear Many-Body Theory of Electroweak Interactions with Nuclei at Intermediate Energies
}

\author{
J. Nieves ${ }^{\text {a }}$ J. E. Amaro ${ }^{\mathrm{a}}$, M. Valverde ${ }^{\mathrm{a}}$ \\ ${ }^{a}$ Departamento de Física Moderna, \\ Universidad de Granada, E-18071 Granada, Spain
}

The Quasi-Elastic (QE) contribution of the nuclear inclusive electron model developed in reference [1] is extended to the study of electroweak Charged Current (CC) induced nuclear reactions at intermediate energies of interest for future neutrino oscillation experiments. Long range nuclear (RPA) correlations, Final State Interaction (FSI) and Coulomb corrections are included within the model. RPA correlations are shown to play a crucial role in the whole range of neutrino energies, up to $500 \mathrm{MeV}$, studied in this work. Predictions for inclusive muon capture for different nuclei through the Periodic Table and for the reactions ${ }^{12} \mathrm{C}\left(\nu_{\mu}, \mu^{-}\right) X$ and ${ }^{12} \mathrm{C}\left(\nu_{e}, e^{-}\right) X$ near threshold are also given.

\section{INTRODUCTION}

Any model aiming at describing the interaction of neutrinos with nuclei should be firstly tested against the existing data on the interaction of real and virtual photons with nuclei. At intermediate energies, nuclear excitation energies ranging from about $100 \mathrm{MeV}$ to 500 or $600 \mathrm{MeV}$, three different contributions should be taken into account: i) QE processes, ii) pion production and two body processes from the $\mathrm{QE}$ region to that beyond the $\Delta(1232)$ resonance peak, and iii) double pion production and higher nucleon resonance degrees of freedom induced processes. The model developed in Refs. 1] (inclusive electro-nuclear reactions) and 2] (inclusive photo-nuclear reactions) has been successfully compared with data at intermediate energies and it systematically includes the three type of contributions mentioned above. Nuclear effects are computed starting from a Local Fermi Gas (LFG) picture of the nucleus, an accurate approximation to deal with inclusive processes which explore the whole nuclear volume [2], and their main features, expansion parameter and all sort of constants are completely fixed from previous hadron-nucleus studies (pionic atoms, elastic and inelastic pion-nucleus reactions, $\Lambda$ - hypernuclei, etc...) 44. Thus, and besides the photon coupling constants determined in the vacuum, the model of Refs. 1] and 2] has no free parameters, and thus the results presented in these two references are predictions deduced from the nuclear microscopic framework developed in Refs. 3] and [4. In this talk, we extent the nuclear inclusive QE electron scattering model of Ref. 1], including the axial CC degrees of freedom, to describe neutrino and antineutrino induced nuclear reactions in the QE region. Detailed formulae and results can be found in [5].

\section{INCLUSIVE CROSS SECTION}

We will expose here the general formalism focusing on the neutrino $\mathrm{CC}$ reaction. The generalization to antineutrino $\mathrm{CC}$ reactions or muon capture is straightforward. In the laboratory frame, the differential cross section for the process $\nu_{l}(k)+A_{Z} \rightarrow l^{-}\left(k^{\prime}\right)+X$ reads:

$\frac{d^{2} \sigma}{d \Omega\left(\hat{k}^{\prime}\right) d E_{l}^{\prime}}=\frac{\left|\vec{k}^{\prime}\right|}{|\vec{k}|} \frac{G^{2}}{4 \pi^{2}} L_{\mu \sigma} W^{\mu \sigma}$

with $L$ and $W$ the leptonic and hadronic tensors, respectively. On the other hand, the inclusive CC nuclear cross section is related to the imaginary

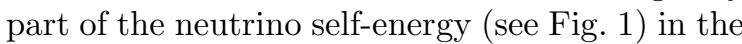


Table 1

Experimental and theoretical flux averaged ${ }^{12} \mathrm{C}\left(\nu_{\mu}, \mu^{-}\right) X$ cross sections. The notation for the theoretical predictions is the same as in Fig. 3

\begin{tabular}{ccccccc}
\hline \multicolumn{3}{c}{ Theory } & \multicolumn{3}{c}{ Exp (LSND) [8] } \\
\hline & LDT & Pauli & RPA & 1995 & 1997 & 2002 \\
\hline $\bar{\sigma}\left[10^{-40} \mathrm{~cm}^{2}\right]$ & 66.1 & 20.7 & 11.9 & $8.3 \pm 0.7 \pm 1.6$ & $11.2 \pm 0.3 \pm 1.8$ & $10.6 \pm 0.3 \pm 1.8$ \\
\hline
\end{tabular}

Table 2

Experimental and theoretical flux averaged ${ }^{12} \mathrm{C}\left(\nu_{e}, e^{-}\right) X$ cross sections. The notation for the theoretical predictions is the same as in Fig. 3

\begin{tabular}{ccccccc}
\hline \multicolumn{5}{c}{ Theory } & \multicolumn{2}{c}{ Exp [9] } \\
\hline & LDT & Pauli & RPA & KARMEN & LSND & LAMPF \\
\hline $\bar{\sigma}\left[10^{-40} \mathrm{~cm}^{2}\right]$ & 5.97 & 0.19 & 0.14 & $0.15 \pm 0.01 \pm 0.01$ & $0.15 \pm 0.01 \pm 0.01$ & $0.141 \pm 0.023$ \\
\hline
\end{tabular}

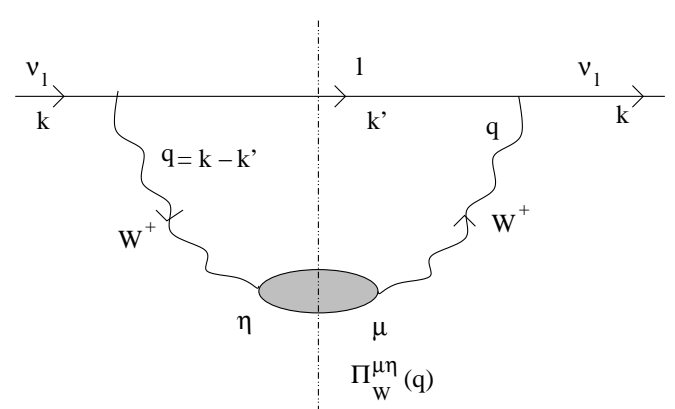

Figure 1. Diagrammatic representation of the neutrino selfenergy in nuclear matter.

medium by:

$\sigma=-\frac{1}{|\vec{k}|} \int \operatorname{Im} \Sigma_{\nu}(k ; \rho(r)) d^{3} r$

We get $\operatorname{Im} \Sigma_{\nu}$ by following the prescription of the Cutkosky's rules: in this case we cut with a vertical straight line (see Fig. 1) the intermediate lepton state and those implied by the $W$-boson polarization. Those states are placed on shell by taking the imaginary part of the propagator, selfenergy, etc. We obtain for $k^{0}>0$

$\operatorname{Im} \Sigma_{\nu}(k)=\frac{8 G \Theta\left(q^{0}\right)}{\sqrt{2} M_{W}^{2}} \int \frac{d^{3} k^{\prime}}{(2 \pi)^{3}} \frac{\operatorname{Im}\left\{\Pi_{W}^{\mu \eta} L_{\eta \mu}\right\}}{2 E_{l}^{\prime}}$

and thus, the hadronic tensor is basically an integral over the nuclear volume of the $W$-selfenergy $\left(\Pi_{W}^{\mu \nu}(q ; \rho)\right)$ inside the nuclear medium. We can then take into account the in-medium effects (such as $W$-absorption by pairs of nucleons, pion production, delta resonances,...) by including the correspondent diagram in the shaded loop of Fig. 1] Further details can be found in Ref. [1].

\section{QE CONTRIBUTION TO $\Pi_{W}^{\mu \nu}(q ; \rho)$}

The virtual $W^{+}$can be absorbed by one nucleon (neutron) leading to the $\mathrm{QE}$ peak of the nuclear response function. Such a contribution corresponds to a $1 \mathrm{p} 1 \mathrm{~h}$ nuclear excitation. We consider a structure of the $V-A$ type for the $W^{+} p n$ vertex, and use PCAC and invariance under G-parity to relate the pseudoscalar form factor to the axial one and to discard a term of the form $\left(p^{\mu}+p^{\prime \mu}\right) \gamma_{5}$ in the axial sector, respectively. Invariance under time reversal guarantees that all form factors are real. Besides, and thanks to isospin symmetry, the vector form factors are related to the electromagnetic ones. We find

$$
\begin{array}{r}
W^{\mu \nu}(q)=\frac{\cos ^{2} \theta_{C}}{2 M^{2}} \int_{0}^{\infty} d r r^{2}\left\{\Theta\left(q^{0}\right) \int \frac{d^{3} p}{4 \pi^{2}} \frac{M}{E(\vec{p})}\right. \\
\frac{M}{E(\vec{p}+\vec{q})} \Theta\left(k_{F}^{n}(r)-|\vec{p}|\right) \Theta\left(|\vec{p}+\vec{q}|-k_{F}^{p}(r)\right) \\
\left.\left.\delta\left(q^{0}+E(\vec{p})-E(\vec{p}+\vec{q})\right) A^{\nu \mu}(p, q)\right|_{p^{0}=E(\vec{p})}\right\}
\end{array}
$$

with the local Fermi momentum $k_{F}(r)=$ $\left(3 \pi^{2} \rho(r) / 2\right)^{1 / 3}, M$ the nucleon mass, and $E(\vec{p})=$ $\sqrt{M^{2}+\vec{p}^{2}}$. We will work on an non-symmetric nuclear matter with different Fermi sea levels 
for protons, $k_{F}^{p}$, than for neutrons, $k_{F}^{n}$ (equation above, but replacing $\rho / 2$ by $\rho_{p}$ or $\rho_{n}$, with $\left.\rho=\rho_{p}+\rho_{n}\right)$. Finally, $A^{\mu \nu}$ is the CC nucleon tensor [5]. The $d^{3} p$ integrations above can be done analytically and all of them are determined by the imaginary part of isospin asymmetric Lindhard function, $\bar{U}\left(q, k_{F}^{n}, k_{F}^{p}\right)$. Explicit expressions can be found in [5].

We take into account polarization effects by substituting the particle-hole (1p1h) response by an RPA response consisting in a series of ph and $\Delta$-hole excitations as shown in Fig. 2 We use an effective Landau-Migdal ph-ph interaction [6]: $V=c_{0}\left\{f_{0}+f_{0}^{\prime} \vec{\tau}_{1} \vec{\tau}_{2}+g_{0} \vec{\sigma}_{1} \vec{\sigma}_{2}+g_{0}^{\prime} \vec{\sigma}_{1} \vec{\sigma}_{2} \vec{\tau}_{1} \vec{\tau}_{2}\right\}$, where only the isovector terms contribute to CC processes. In the $S=1=T$ channel $(\vec{\sigma} \vec{\sigma} \vec{\tau} \vec{\tau}$ operator) we use an interaction 124 with explicit $\pi$ (longitudinal) and $\rho$ (transverse) exchanges. The $\Delta(1232)$ degrees of freedom are also included in the $S=1=T$ channel of the RPA response. This effective interaction is non-relativistic, and then for consistency we will neglect terms of or$\operatorname{der} \mathcal{O}\left(p^{2} / M^{2}\right)$ when summing up the RPA series.

We ensure the correct energy balance, of both neutrino and antineutrino CC induced process in finite nuclei, by modifying the energy conserving $\delta$ function in Eq. (4) to account for the $Q$-value of the reaction.

We also consider the effect of the Coulomb field of the nucleus acting on the ejected charged lepton. This is done by including the lepton selfenergy $\Sigma_{C}=2 k^{\prime 0} V_{C}(r)$ in the intermediate lepton propagator of Fig. [1]

Finally, we account for the FSI by using nucleon propagators properly dressed with a realistic self-energy in the medium, which depends explicitly on the energy and the momentum [7]. Thus, we rewrite the imaginary part of the Lindhard function (ph propagator) in terms of spectral functions $S_{p, h}(\omega, \vec{p} ; \rho)$.

\section{RESULTS}

We present in Fig. [3 and Tables 1 and 2 our theoretical predictions and a comparison of those to the experimental measurements of the inclusive ${ }^{12} \mathrm{C}\left(\nu_{\mu}, \mu^{-}\right) X$ and ${ }^{12} \mathrm{C}\left(\nu_{e}, e^{-}\right) X$ reactions

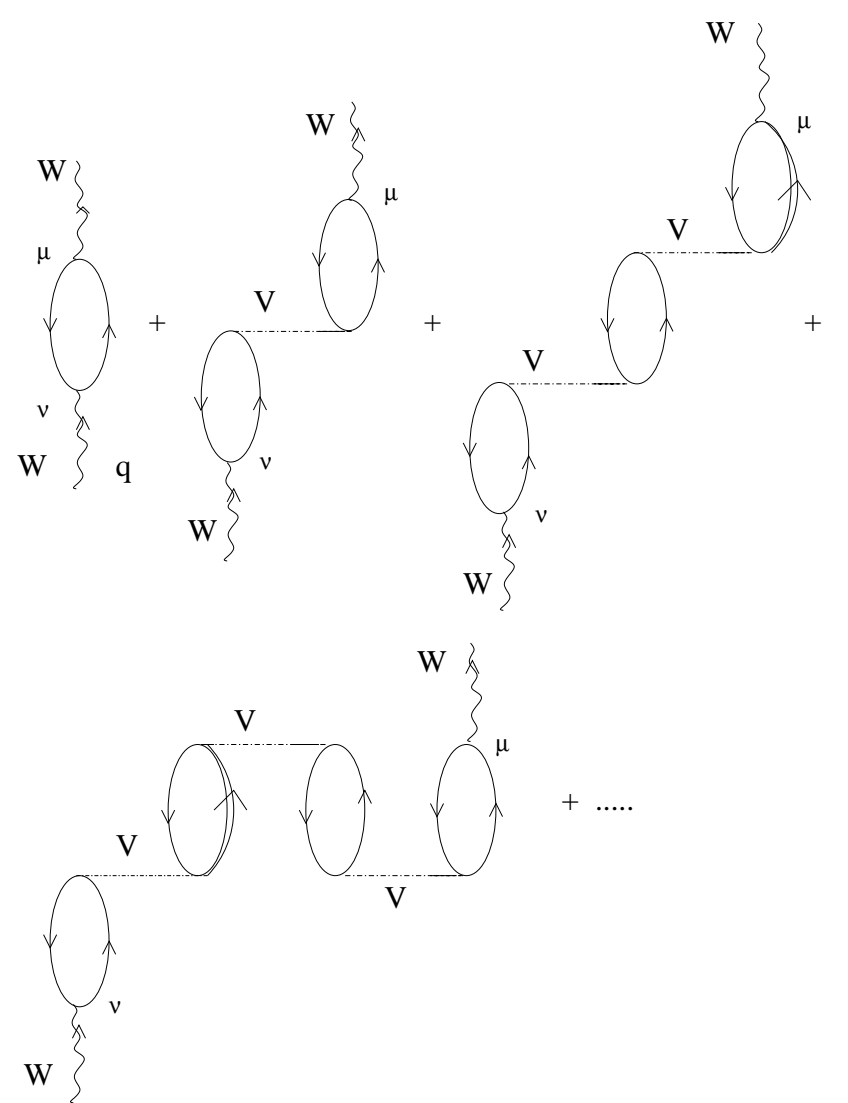

Figure 2. Set of irreducible diagrams responsible for the polarization (RPA) effects in the $1 \mathrm{p} 1 \mathrm{~h}$ contribution to the $W$-selfenergy.

near threshold. Pauli blocking and the use of the correct energy balance improve the results, but only once RPA and Coulomb effects are included a good description of data is achieved.

Given the success in describing the LSND measurement of the reaction ${ }^{12} \mathrm{C}\left(\nu_{\mu}, \mu^{-}\right) X$ near threshold, it seems natural to further test our model by studying the closely related process of the inclusive muon capture in ${ }^{12} \mathrm{C}$. Furthermore, and since there is abundant and accurate measurements on nuclear inclusive muon capture rates through the whole Periodic Table, we have also calculated muon capture widths for a few selected nuclei. Results are compiled in Table 3 Data are quite accurate, with precisions smaller than $1 \%$, quite far from the theoretical uncertain- 

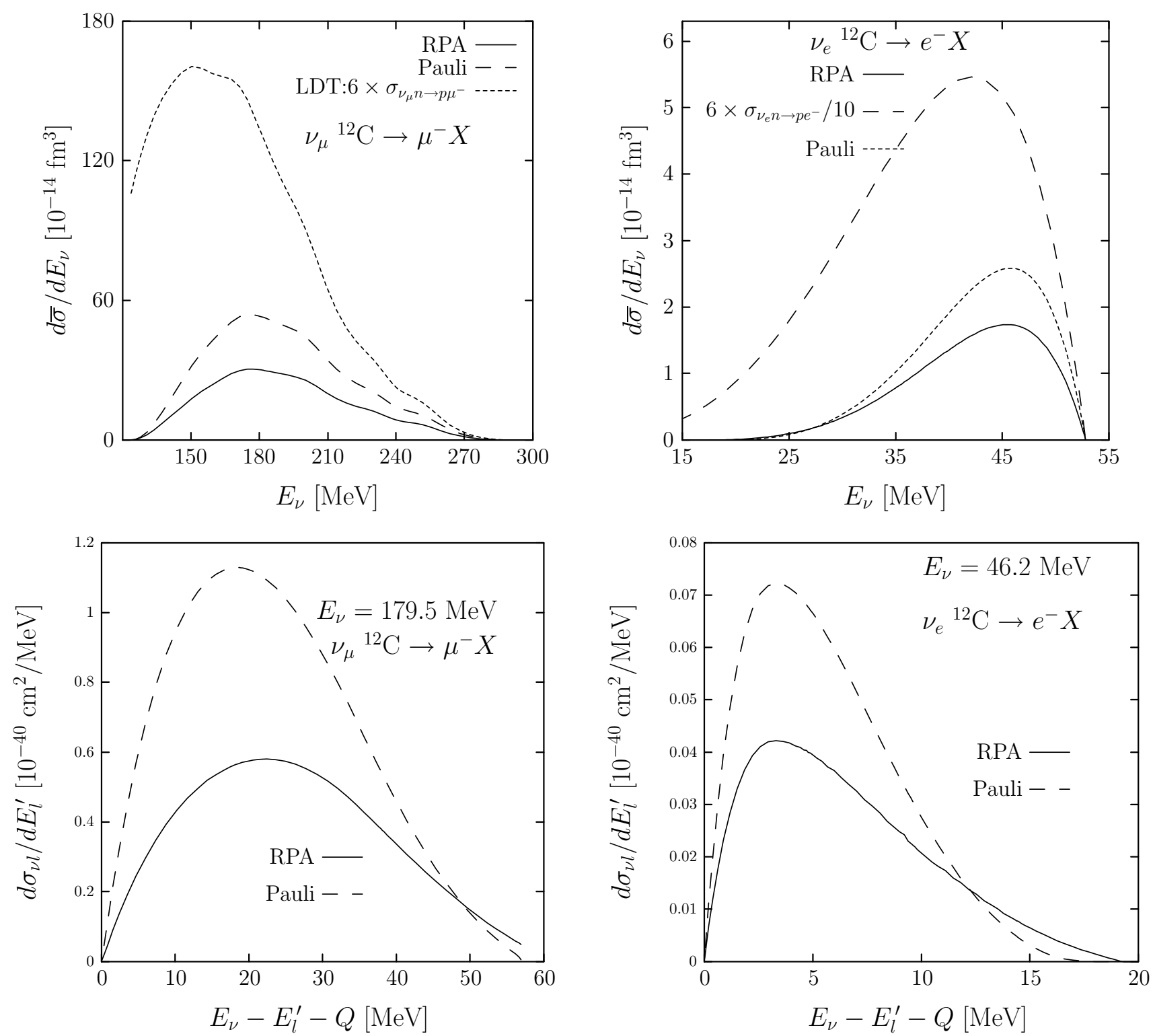

Figure 3. Theoretical predictions for the ${ }^{12} \mathrm{C}\left(\nu_{\mu}, \mu^{-}\right) X$ and the ${ }^{12} \mathrm{C}\left(\nu_{e}, e^{-}\right) X$ reactions near threshold. In addition to the full calculation (denoted as RPA), with all nuclear effects with the exception of the FSI ones, we show results obtained without including RPA, FSI and Coulomb corrections (denoted as Pauli), and also results (denoted as LDT) obtained by multiplying the free space cross section by the number of neutrons of ${ }^{12} \mathrm{C}$. 
Table 4

Electron neutrino (left) and antineutrino (right) inclusive QE integrated cross sections from oxygen. We present results for relativistic ('REL') and non-relativistic nucleon kinematics. In this latter case, we present results with ('FSI') and without ('NOREL') FSI effects. Results, denoted as 'RPA' and 'Pauli' have been obtained with and without including RPA correlations and Coulomb corrections, respectively. Units: $10^{-40} \mathrm{~cm}^{2}$.

\begin{tabular}{clcccccc}
\hline$E_{\nu}[\mathrm{MeV}]$ & \multicolumn{3}{c}{$\sigma\left({ }^{16} \mathrm{O}\left(\nu_{e}, e^{-} X\right)\right)$} & \multicolumn{3}{c}{$\sigma\left({ }^{16} \mathrm{O}\left(\bar{\nu}_{e}, e^{+} X\right)\right)$} \\
\hline \multirow{2}{*}{400} & & REL & NOREL & FSI & REL & NOREL & FSI \\
& Pauli & 389.4 & 416.6 & 352.5 & 130.0 & 139.1 & 121.0 \\
& RPA & 294.7 & 322.6 & 303.6 & 91.9 & 101.9 & 104.8 \\
\hline \multirow{2}{*}{310} & Pauli & 281.4 & 297.4 & 240.6 & 98.1 & 104.0 & 87.2 \\
& RPA & 192.2 & 209.0 & 195.2 & 65.9 & 72.4 & 73.0 \\
\hline \multirow{2}{*}{220} & Pauli & 149.5 & 156.2 & 121.2 & 60.7 & 63.6 & 51.0 \\
& RPA & 90.1 & 97.3 & 92.8 & 36.8 & 40.0 & 40.2 \\
\hline
\end{tabular}

Table 3

Experimental and theoretical total muon capture widths for different nuclei, in $\left[10^{4} \mathrm{~s}^{-1}\right]$ units. Data are taken from Ref. 10, using a weighted average: $\bar{\Gamma} / \sigma^{2}=\sum_{i} \Gamma_{i} / \sigma_{i}^{2}$, with $1 / \sigma^{2}=\sum_{i} 1 / \sigma_{i}^{2}$. We quote two different theoretical results: with (RPA) and without (Pauli) the inclusion of RPA correlations. FSI effects are not included in any of them.

\begin{tabular}{lccc}
\hline & Pauli & RPA & Exp \\
\hline${ }^{12} \mathrm{C}$ & 5.42 & 3.21 & $3.78 \pm 0.03$ \\
${ }^{16} \mathrm{O}$ & 17.56 & 10.41 & $10.24 \pm 0.06$ \\
${ }^{18} \mathrm{O}$ & 11.94 & 7.77 & $8.80 \pm 0.15$ \\
${ }^{23} \mathrm{Na}$ & 58.38 & 35.03 & $37.73 \pm 0.14$ \\
${ }^{40} \mathrm{Ca}$ & 465.5 & 257.9 & $252.5 \pm 0.6$ \\
${ }^{44} \mathrm{Ca}$ & 318 & 189 & $179 \pm 4$ \\
${ }^{75} \mathrm{As}$ & 1148 & 679 & $609 \pm 4$ \\
${ }^{112} \mathrm{Cd}$ & 1825 & 1078 & $1061 \pm 9$ \\
${ }^{208} \mathrm{~Pb}$ & 1939 & 1310 & $1311 \pm 8$ \\
\hline
\end{tabular}

ties of any existing model. Medium polarization effects (RPA correlations), once more, are essential to describe the data. Despite of the huge range of variation of the capture widths (note, $\Gamma^{\exp }$ varies from about $4 \times 10^{4} \mathrm{~s}^{-1}$ in ${ }^{12} \mathrm{C}$ to 1300 $\times 10^{4} \mathrm{~s}^{-1}$ in ${ }^{208} \mathrm{~Pb}$ ), the agreement to data is quite good for all studied nuclei, with discrepancies of about $15 \%$ at most. It is precisely for ${ }^{12} \mathrm{C}$, where we find the greatest discrepancy with experiment. Nevertheless, our model provides one of the best existing combined description of the inclusive muon capture in ${ }^{12} \mathrm{C}$ and the LSND measurement of the reaction ${ }^{12} \mathrm{C}\left(\nu_{\mu}, \mu^{-}\right) X$ near threshold.

At intermediate energies the predictions of our model become reliable not only for integrated cross sections, but also for differential cross sections. We present results for incoming neutrino energies within the interval 150-400 (250-500) $\mathrm{MeV}$ for electron (muon) species. In Figs. 4and 5 FSI effects on differential cross section are shown. As expected, FSI provides a broadening and a significant reduction of the strength of the QE peak. Nevertheless the integrated cross section is only slightly modified. In Table 4 we compile electron neutrino and antineutrino inclusive QE integrated cross sections from oxygen. Though FSI change importantly the differential cross sections, it plays a minor role when one considers total cross sections. When medium polarization effects are not considered, FSI provides significant reductions (13-29\%) of the cross sections. However, when RPA corrections are included the reductions becomes more moderate, always smaller than $7 \%$, and even there exist some cases where FSI enhances the cross sections. This can be easily understood by looking at Fig. 5 There, we see that FSI increases the cross section for high energy transfer. But for nuclear excitation energies higher than those around the QE peak, the RPA corrections are certainly less important than in the peak region. Hence, the RPA suppression of the FSI distribution is significantly smaller than 

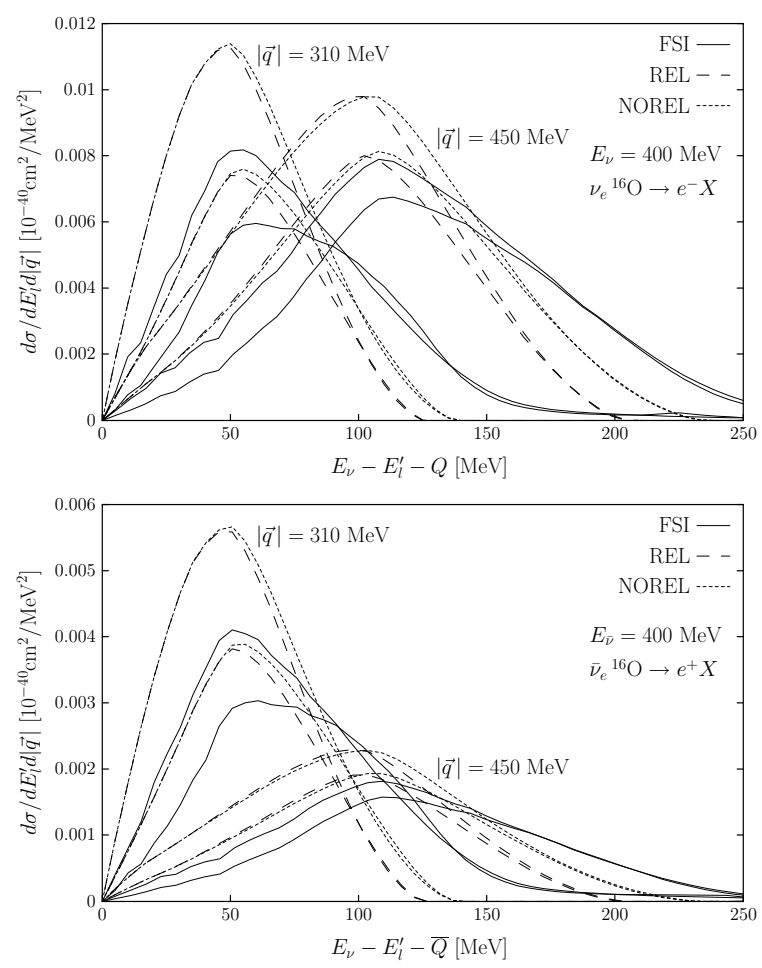

Figure 4. $\nu_{e}-$ (top) and $\bar{\nu}_{e}-$ (bottom) inclusive QE differential cross sections in oxygen as a function of the transferred energy, at two values (310 and $450 \mathrm{MeV}$ ) of the transferred momentum. The incoming neutrino (antineutrino) energy is $400 \mathrm{MeV}$. We show results for relativistic (REL) and non-relativistic nucleon kinematics. In this latter case, we present results with (FSI) and without (NOREL) FSI effects. For the three cases, we also show the effect of taking into account RPA correlations and Coulomb corrections (lower lines at the peak).

the RPA reduction of the distribution determined by the ordinary Lindhard function.

\section{REFERENCES}

1. A. Gil, J. Nieves and E. Oset, Nucl. Phys. A627 (1997) 543; ibidem Nucl. Phys. A627 (1997) 598.

2. R.C. Carrasco and E. Oset, Nucl. Phys. A536 (1992) 445; R.C. Carrasco, E. Oset and L.L. Salcedo, Nucl. Phys. A541 (1992) 585; R.C. Carrasco, M.J. Vicente-Vacas and E. Oset, Nucl. Phys. A570 (1994) 701.

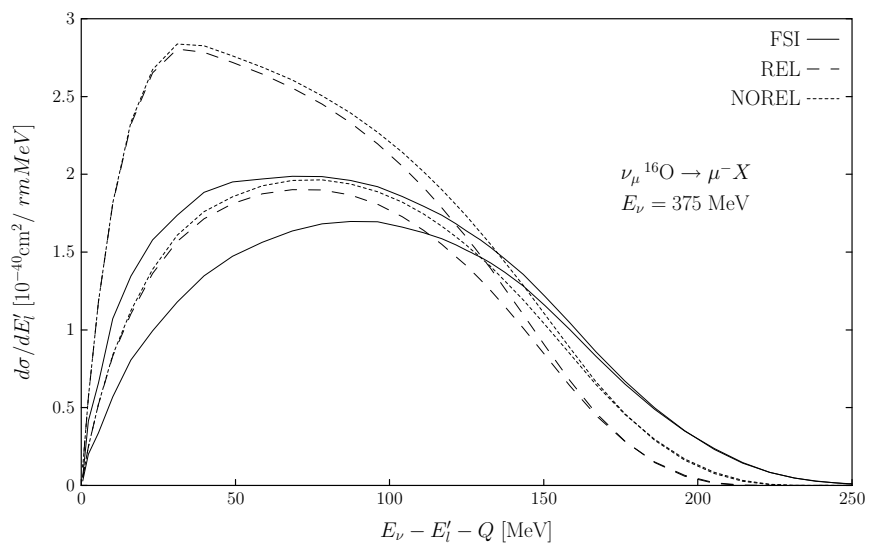

Figure 5. Muon neutrino inclusive QE differential cross sections in oxygen as a function of the transferred energy. The incoming neutrino energy is $375 \mathrm{MeV}$. The notation for the theoretical predictions is the same as in Fig. 4

3. E. Oset, H. Toki and W. Weise, Phys. Rep. 83 (1982) 281.

4. J. Nieves, E. Oset, C. García-Recio, Nucl. Phys. A554 (1993) 509; ibidem Nucl. Phys. A554 (1993) 554; E. Oset, et al., Prog. Theor. Phys. Suppl. 117 (1994) 461; C. Albertus, J.E. Amaro and J. Nieves, Phys. Rev. Lett. 89 (2002) 032501; ibidem Phys. Rev. C67 (2003) 034604 .

5. J. Nieves, J.E. Amaro and M. Valverde, nucl-th/0408005

6. J. Speth, E. Werner and W. Wild, Phys. Rep. 33 (1977) 127; J. Speth, V. Klemt, J. Wambach and G.E. Brown Nucl. Phys. A343 (1980) 382.

7. P. Fernández de Córdoba and E. Oset, Phys. Rev. C46 (1992) 1697.

8. M. Albert, et al., Phys. Rev. C51 (1995) R1065; C. Athanassopoulos, et al., Phys. Rev. C56 (1997) 2806; L.B. Auerbach, et al., Phys. Rev. C66 (2002) 015501.

9. B. Zeitnitz, Prog. Part. Nucl. Phys. 32 (1994) 351; B.E. Bodmann et al., Phys. Lett. B332 (1994) 251; C. Athanassopoulos, et al., Phys. Rev. C55 (1997) 2078; D.A. Krakauer et al., Phys. Rev. C45 (1992) 2450.

10. T. Suzuki, D.F. Measday and J.P. Roalsvig, Phys. Rev. C35 (1987) 2212. 